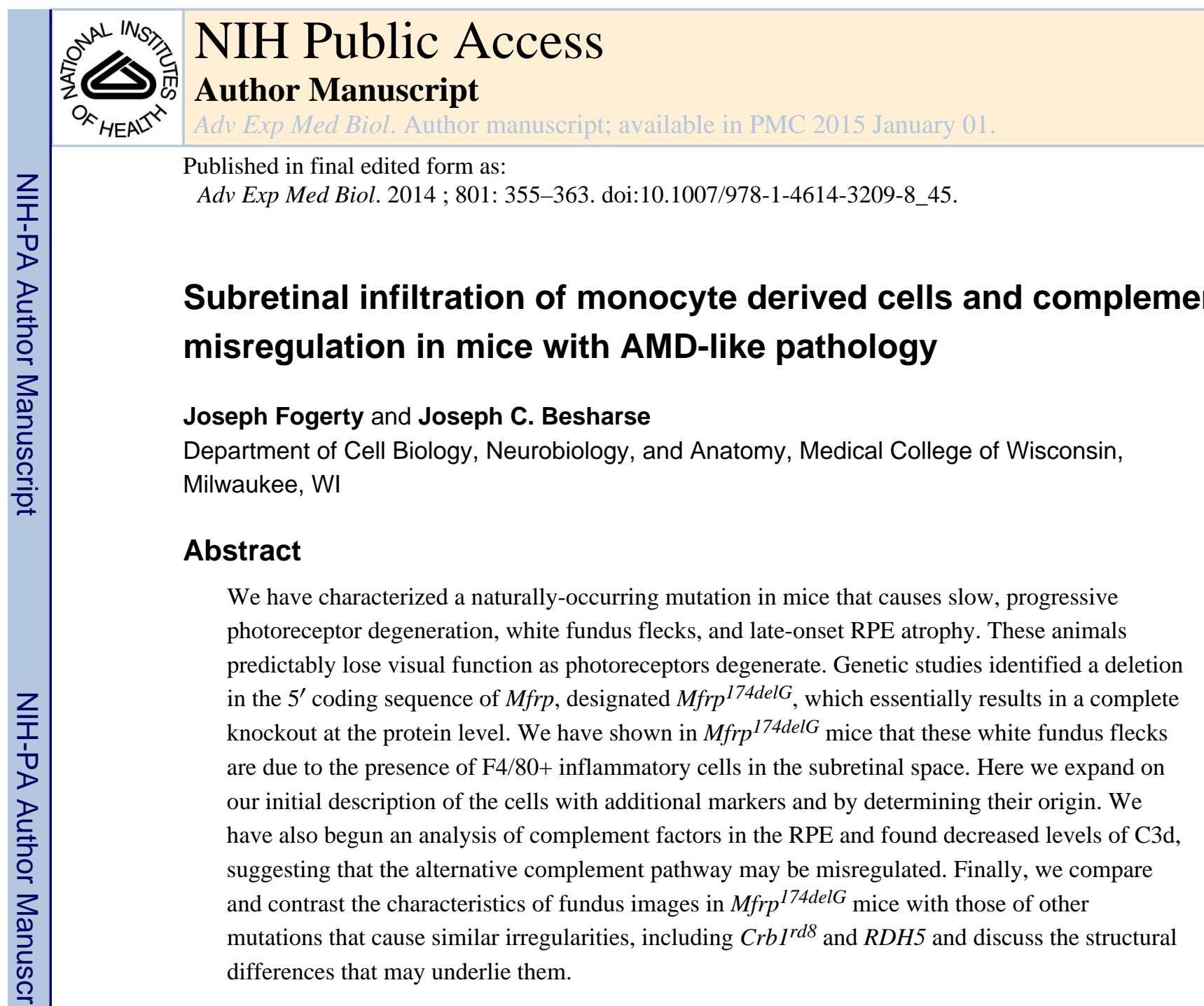

\title{
45.1 Introduction
}

We have previously described a novel mutation in mouse Mfrp that results in AMD-like symptoms [1]. These animals have a dramatic, late-onset RPE degeneration phenotype that looks similar to human GA. Although mice do not have a macula, it is possible that the patchwork atrophy that we reported in $M$ frp $^{174 d e l G}$ mutants simulates similar loss of RPE in humans and that these animals could provide an attractive model.

An additional feature of $M$ frp $^{174 d e l G}$ mice is a regular array of white retinal flecks that is seen in fundus images during the period of photoreceptor loss[1]. Our earlier work established that these white flecks were likely macrophages infiltrating the subretinal space. We expand on that analysis here by showing that the white flecks represent individual cells that express several markers indicative of a macrophage/monocyte lineage and further show in bone marrow chimeras that these macrophages are derived from circulating monocytes. We also report on an initial analysis of key complement regulators and discuss possible contributions this pathway may make to disease progression. Finally, we compare and contrast the "white fleck" phenotype seen in $M$ frp ${ }^{174 d e l G}$ mice with fundus abnormalities seen in $\mathrm{Crbl}^{\mathrm{rd} 8}$ mice, and discuss the possible structural basis for these observations.

Correpsonding Author: Joseph C. Besharse, 8701 Watertown Plank Rd, BSB473, Milwaukee, WI 53226; Phone: (414) 955-8261; jbeshars@mcw.edu. 


\subsection{Materials and Methods}

\subsubsection{Imaging and Image Analysis}

Animals were killed with $\mathrm{CO}_{2}$ and eyes were dissected under a Leica MZFLIII microscope. The cornea and lens were removed with fine scissors and the retina was gently peeled away. The remaining eyecup was then fixed in PBS containing $4 \%$ paraformaldehyde. Images of the apical RPE surface were collected with a digital camera that was mounted on the dissecting microscope. The tissue was then stained with Hoescht, flat mounted under a coverslip, and nuclei from subretinal cells adherent to the apical RPE were imaged with a Nikon TE300 microscope and a Coolsnap HQ camera. The coordinates of each spot (in the dissecting scope image) or nucleus (in the fluorescence image) were determined, and the average nearest neighbor distance was calculated from each image.

\subsubsection{Immunohistochemistry}

Antibodies used were as follows: GFP (Torrey Pines, 1:250), Cd11b-FITC (Life Technologies, 1:250), CD68 (Millipore, 1:100), Iba1 (Wako, 1:1000), GS-IB4-488 (Life Technologies, 1:50). Fixed eyecups (see above) were permeabilized in PBS $+0.1 \%$ Triton $\mathrm{X}-100$ for 2 minutes, blocked 1 hour in PBS $+1 \%$ BSA, and incubated in the appropriate antibody overnight at $4{ }^{\circ} \mathrm{C}$. Tissue was then washed three times in PBS, incubated 30 minutes in the appropriate Alexa-488-conjugated secondary antibody, and counterstained with Hoescht (see above). The autofluorescence signal was collected using a long-pass rhodamine filter set. For analysis of $\mathrm{C} 3 \mathrm{~d}$, eyecups were fixed in $2 \%$ paraformaldehyde, soaked in $50 \%$ sucrose, and embedded in OCT. 10um sections were permeabilized, blocked as above and probed with C3d antibody (R\&D Systems, 1:100).

\subsubsection{Bone Marrow Transplant}

ROSA26-YFP reporter mice [2] were bred to EIIa-Cre[3] mice to create a line of YFP+ mice ubiquitously expressing YFP. Bone marrow from these animals was then transplanted into lethally irradiated weanling $M \mathrm{frp}^{174 d e l G}$ mice. The resulting bone marrow chimeras were then examined 8 weeks later ( 3 months of age) when the subretinal cells in significant numbers are first apparent in fundus images. Cells were immunostained and viewed in whole mounts using a GFP antibody (see above).

\subsubsection{Immunoblotting}

RPE-choroid was scraped off of eyecups (see above), sonicated directly in Laemmli buffer and western blotted using CFH and CFB antibodies (Quidel, each at 1:5000). Blots were visualized and quantitatively analyzed relative to endogenous actin with a Li-Cor Odyssey infrared detection system.

\subsection{Results}

\subsubsection{White spots seen in fundus images of Mfrp ${ }^{174 d e l G}$ mice represent cells in the subretinal space}

It has been noted previously that $M f^{r p}{ }^{174 d e l G}$ mice exhibit white flecks visible by fundus imaging that correspond to cells in the subretinal space $[1,4-5]$. A Mfrp ${ }^{174 d e l G}$ mouse in 
which retinal flecks were observed by fundus photography was used to test that conclusion with more deliberate measurements. The retina was removed from the eyecup and the white spots on the RPE were directly imaged (Figure 1A), and the mean distance from each spot to its nearest neighbor was determined to be $44.5 \mu \mathrm{m}$ (I lost the standard error measure. Probably should put it back!). The same specimen was then flattened, stained with Hoescht and imaged by fluorescence microscopy (Figure 1B). The difference in focal plane between the subretinal cells and the underlying RPE, combined with the dense pigmentation of the RPE, allowed us to easily distinguish the nuclei of the two cell populations. The nearestneighbor distance for the Hoescht-stained subretinal cells was 47.4 $\mu \mathrm{m}$ (standard error!). These data are consistent with the conclusion that the white spots correspond 1 to 1 with subretinal cells.

\subsubsection{Subretinal cells express markers characteristic of macrophages and are derived directly from circulating monocytes}

To further phenotype the subretinal cells, we immunostained them for markers of monocytederived cells including Iba1, CD11b, CD68 (ED-1), and the lectin GS-IB4 [6-7] in separate preparations. Iba1, CD11b, and CD68 antibodies labeled all of the subretinal cells in their respective preparations, while GS-IB4 labeled only a subset of them (Figure 2). We identified the origin of the subretinal macrophages by making bone marrow chimeras with YFP+ donor cells. Of $8 \mathrm{Mfrp}{ }^{174 d e l G}$ bone marrow chimeras examined in detail, the subretinal macrophages were overwhelmingly YFP+ (Figure 3A-B) and therefore derived from donor's bone marrow. Only a single subretinal cell among hundreds examined was not unequivocally YFP+. Furthermore, the chimeric animals, which had $M f r p^{+/+}$subretinal macrophages, did not exhibit any change in degree of retinal degeneration when compared to age-matched, unaltered $M f r p{ }^{174 d e l G}$ mice (Figure 3C), suggesting that the $M$ frp ${ }^{174 d e l G}$ monocyte-derived cells are not primarily responsible for photoreceptor loss.

\subsubsection{Evidence of depressed activity of the alternative complement pathway}

Since it is widely hypothesized that the complement system may be involved in AMD pathogenesis, we evaluated two candidate components of the alternative complement pathway. Western blotting of mouse RPE+choroid in weanling animals showed that factors B and H were consistently decreased in Mfrp ${ }^{174 d e l G}$ animals. We also evaluated C3d abundance in frozen sections from 5 month old animals and found it to be similarly decreased (Figure 4).

\subsubsection{Comparison of fundus images of Mfrp ${ }^{174 d e l G}$ and Crb1 ${ }^{\text {rd8 }}$ mice}

Comparison of $M \mathrm{frp}^{174 d e l G}$ and $\mathrm{Crb}^{\mathrm{rd} 8}$ mice illustrate the limitations of fundus imaging for delineating individual cells without additional correlative analysis. The $r d 8$ phenotype [8] includes white fundus spots which are typically large and irregular, but can sometimes be smaller and more discrete (Figure 5A-B). Some of these could easily be mistaken for the white flecks we have observed in $M f r p^{174 d e l G}$ animals. However, the lesions in $r d 8$ mice are due to retinal folds that protrude into and distort the outer nuclear layer [8]. The lighter appearance of the fundus in these affected areas may be due to increased reflectance from accumulations of inner and outer segment material within the retinal folds. Alternatively, it 
may be due to changes in light scattering properties of the outer retina due to disruption of the ONL [9]. White flecks corresponding to subretinal macrophages in Mfrp ${ }^{174 d e l G}$ mice, however, are consistently uniform and small in size, and while they can be sparse at early time points, they eventually cover the entire fundus with a remarkably uniform distribution. Interestingly, mutations in $R D H 5$ and $R L B P 1$ produce fundus images in humans with discrete white spots, similar to what we see in $M$ frp $^{174 d e l G}$ mice [10-11]. In addition, Rep1 mutant mice have autofluorescent retinal spots that can be seen with $488 \mathrm{~nm}$ SLO as well as subretinal cells [12]. To our knowledge these observations have never been correlated with histological sections, although OCT scans yield results that are consistent with cells in the subretinal space [13-15].

\subsection{Discussion}

We have expanded on our previous analysis of the $M$ frp ${ }^{174 d e l G}$ mouse and have found additional evidence of immune system involvement. More precise correlation of the white fundus flecks and the subretinal cells cements the presumption that they are the same objects. It also provides evidence that refraction caused by a single cell is not beyond the resolution of a standard fundus camera. Furthermore, we now show that these cells express additional markers characteristic of macrophages. The non-uniformity of GS-IB4 reactivity suggests that there is some degree of phenotypic heterogeneity among these cells, possibly reflecting variable degrees of cell activation [16]. We note that there is currently no clear consensus on markers that distinguish between various types of monocyte-derived cells, and that different laboratories have established different phenotyping criteria. Indeed, it has even been suggested that such a marker may not exist [17-18].

The origin of these subretinal cells is a particular point of interest. $C x 3 \mathrm{crl}$ mutant animals exhibit over-accumulation of subretinal Iba1+ cells, which appear to be derived from resident microglia that migrate down from the inner retina [19]. This is also a normal occurrence in Balb/c mice under standard lighting conditions [20]. We entertained the alternate hypothesis that the subretinal cells in $M$ frpp ${ }^{174 d e l G}$ mice were derived directly from circulating monocytes. Bone marrow chimeras made with YFP+ donor cells clearly showed that these cells were indeed derived from the donor marrow. We considered the possibility that the donor cells might first engraft in the retina where they would mix with host microglia and then migrate to the subretinal space. If that were the case, however, we would have expected a more mixed population of YFP+ donor cells and YFP- host retinal microglial cells. The fact that they were essentially all donor-derived suggests that they arrive at this site via a more direct route.

The complement pathway has been of interest in AMD ever since the discovery that polymorphisms in key complement regulation genes are significant risk factors for developing the disease[21-22]. Factor B is a key positive regulator of the pathway, while factor $\mathrm{H}$ is a negative regulator; we found decreased levels of both in $M$ frp ${ }^{174 d e l G} \mathrm{RPE}$ +choroid. When the alternative pathway is activated, large amounts of $\mathrm{C} 3 \mathrm{~b}$ are produced, but the half-life of $\mathrm{C} 3 \mathrm{~b}$ is relatively short and it is rapidly cleaved to generate C3d (among other fragments), which then accumulates at the site [23]. The observation of decreases in both CFH and CFB is somewhat contradictory, but the decrease in C3d as well suggests a 
net depression in complement activation. The extent to which these changes may be associated with pathology in $M f r p^{174 d e l G}$ remains to be investigated. However, there is recent evidence that the disruption of baseline complement signaling results in photoreceptor degeneration [24].

\section{References}

1. Fogerty J, Besharse JC. 174delG mutation in mouse MFRP causes photoreceptor degeneration and RPE atrophy. Invest Ophthalmol Vis Sci. 2011; 52(10):7256-66. [PubMed: 21810984]

2. Srinivas S, Watanabe T, Lin CS, William CM, Tanabe Y, Jessell TM, Costantini F. Cre reporter strains produced by targeted insertion of EYFP and ECFP into the ROSA26 locus. BMC Dev Biol. 2001; 1:4. [PubMed: 11299042]

3. Lakso M, Pichel JG, Gorman JR, Sauer B, Okamoto Y, Lee E, Alt FW, Westphal H. Efficient in vivo manipulation of mouse genomic sequences at the zygote stage. Proc Natl Acad Sci U S A. 1996; 93(12):5860-5. [PubMed: 8650183]

4. Hawes NL, Chang B, Hageman GS, Nusinowitz S, Nishina PM, Schneider BS, Smith RS, Roderick TH, Davisson MT, Heckenlively JR. Retinal degeneration 6 (rd6): a new mouse model for human retinitis punctata albescens. Invest Ophthalmol Vis Sci. 2000; 41(10):3149-57. [PubMed: 10967077]

5. Kameya S, Hawes NL, Chang B, Heckenlively JR, Naggert JK, Nishina PM. Mfrp, a gene encoding a frizzled related protein, is mutated in the mouse retinal degeneration 6. Hum Mol Genet. 2002; 11(16):1879-86. [PubMed: 12140190]

6. Joly S, Francke M, Ulbricht E, Beck S, Seeliger M, Hirrlinger P, Hirrlinger J, Lang KS, Zinkernagel M, Odermatt B, Samardzija M, Reichenbach A, Grimm C, Reme CE. Cooperative phagocytes: resident microglia and bone marrow immigrants remove dead photoreceptors in retinal lesions. Am J Pathol. 2009; 174(6):2310-23. [PubMed: 19435787]

7. Rutar MV, Natoli RC, Provis JM. Small interfering RNA-mediated suppression of Ccl2 in Muller cells attenuates microglial recruitment and photoreceptor death following retinal degeneration. $\mathrm{J}$ Neuroinflammation. 2012; 9(1):221. [PubMed: 22992301]

8. Mehalow AK, Kameya S, Smith RS, Hawes NL, Denegre JM, Young JA, Bechtold L, Haider NB, Tepass U, Heckenlively JR, Chang B, Naggert JK, Nishina PM. CRB1 is essential for external limiting membrane integrity and photoreceptor morphogenesis in the mammalian retina. Hum Mol Genet. 2003; 12(17):2179-89. [PubMed: 12915475]

9. Solovei I, Kreysing M, Lanctot C, Kosem S, Peichl L, Cremer T, Guck J, Joffe B. Nuclear architecture of rod photoreceptor cells adapts to vision in mammalian evolution. Cell. 2009; 137(2): 356-68. [PubMed: 19379699]

10. Yamamoto H, Simon A, Eriksson U, Harris E, Berson EL, Dryja TP. Mutations in the gene encoding 11-cis retinol dehydrogenase cause delayed dark adaptation and fundus albipunctatus. Nat Genet. 1999; 22(2):188-91. [PubMed: 10369264]

11. Demirci FY, Rigatti BW, Mah TS, Gorin MB. A novel compound heterozygous mutation in the cellular retinaldehyde-binding protein gene (RLBP1) in a patient with retinitis punctata albescens. Am J Ophthalmol. 2004; 138(1):171-3. [PubMed: 15234312]

12. Tolmachova T, Anders R, Abrink M, Bugeon L, Dallman MJ, Futter CE, Ramalho JS, Tonagel F, Tanimoto N, Seeliger MW, Huxley C, Seabra MC. Independent degeneration of photoreceptors and retinal pigment epithelium in conditional knockout mouse models of choroideremia. J Clin Invest. 2006; 116(2):386-94. [PubMed: 16410831]

13. Wang NK, Chuang LH, Lai CC, Chou CL, Chu HY, Yeung L, Chen YP, Chen KJ, Wu WC, Chen TL, Chao AN, Hwang YS. Multimodal fundus imaging in fundus albipunctatus with RDH5 mutation: a newly identified compound heterozygous mutation and review of the literature. Doc Ophthalmol. 2012; 125(1):51-62. [PubMed: 22669287]

14. Querques G, Carrillo P, Querques L, Bux AV, Del Curatolo MV, Delle Noci N. High-definition optical coherence tomographic visualization of photoreceptor layer and retinal flecks in fundus albipunctatus associated with cone dystrophy. Arch Ophthalmol. 2009; 127(5):703-6. [PubMed: 19433727] 
15. Schatz P, Preising M, Lorenz B, Sander B, Larsen M, Eckstein C, Rosenberg T. Lack of autofluorescence in fundus albipunctatus associated with mutations in RDH5. Retina. 2010; 30(10):1704-13. [PubMed: 20829743]

16. Tabor DR, Theus SA, Barnett JB, Tryka AF, Jacobs RF. The concurrent expression of Griffonia simplicifolia-IB4 binding and tumor necrosis factor-alpha differs between alveolar and peritoneal macrophages. Proc Soc Exp Biol Med. 1992; 199(3):351-9. [PubMed: 1539038]

17. Schmid CD, Melchior B, Masek K, Puntambekar SS, Danielson PE, Lo DD, Sutcliffe JG, Carson MJ. Differential gene expression in LPS/IFNgamma activated microglia and macrophages: in vitro versus in vivo. J Neurochem. 2009; 109 (Suppl 1):117-25. [PubMed: 19393017]

18. Graeber MB, Streit WJ. Microglia: biology and pathology. Acta Neuropathol. 2010; 119(1):89105. [PubMed: 20012873]

19. Kezic J, McMenamin PG. Differential turnover rates of monocyte-derived cells in varied ocular tissue microenvironments. J Leukoc Biol. 2008; 84(3):721-9. [PubMed: 18577714]

20. Ng TF, Streilein JW. Light-induced migration of retinal microglia into the subretinal space. Invest Ophthalmol Vis Sci. 2001; 42(13):3301-10. [PubMed: 11726637]

21. Daiger SP. Genetics. Was the Human Genome Project worth the effort? Science. 2005; 308(5720): 362-4. [PubMed: 15769856]

22. Gold B, Merriam JE, Zernant J, Hancox LS, Taiber AJ, Gehrs K, Cramer K, Neel J, Bergeron J, Barile GR, Smith RT, Hageman GS, Dean M, Allikmets R. Variation in factor B (BF) and complement component 2 (C2) genes is associated with age-related macular degeneration. Nat Genet. 2006; 38(4):458-62. [PubMed: 16518403]

23. Scholl HP, Charbel Issa P, Walier M, Janzer S, Pollok-Kopp B, Borncke F, Fritsche LG, Chong NV, Fimmers R, Wienker T, Holz FG, Weber BH, Oppermann M. Systemic complement activation in age-related macular degeneration. PLoS One. 2008; 3(7):e2593. [PubMed: 18596911]

24. Yu M, Zou W, Peachey NS, McIntyre TM, Liu J. A novel role of complement in retinal degeneration. Invest Ophthalmol Vis Sci. 2012; 53(12):7684-92. [PubMed: 23074214] 

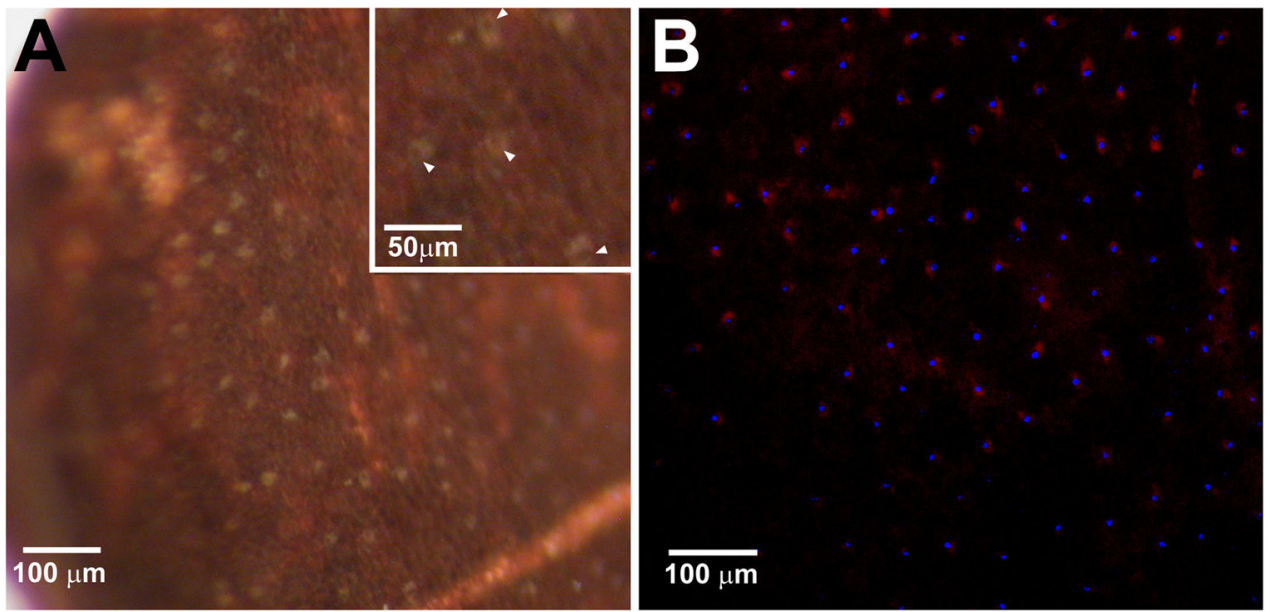

Figure 1. Correlation of fundus spots with cells in the subretinal space

(A) White spots were imaged under a dissecting microscope. Many of these spots had "holes" in their center, suggestive of a nucleus (arrows, insert). (B) The same piece of tissue was stained with Hoescht and flat mounted (blue = nuclei, red = autofluorescence). 


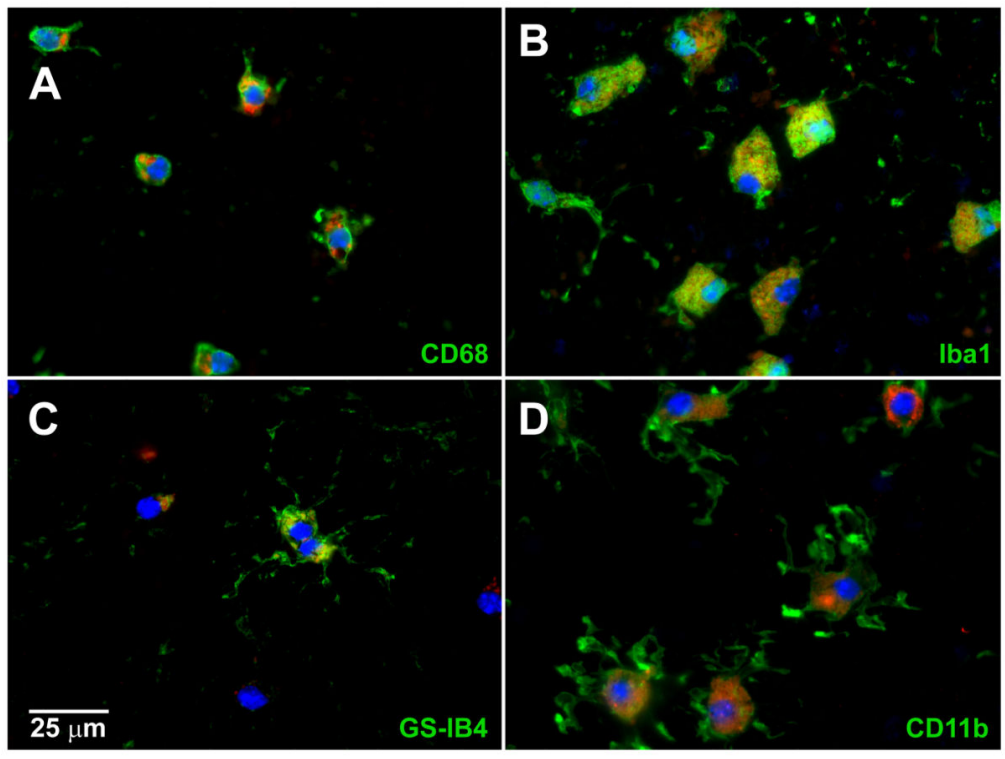

Figure 2. Immunostaining with markers of monocyte-derived cells

Subretinal cells in whole mounts all stained positive for markers of monocyte derived cells including CD11b, Iba1, and CD68. A fraction of the cells were also GS-IB4+. 

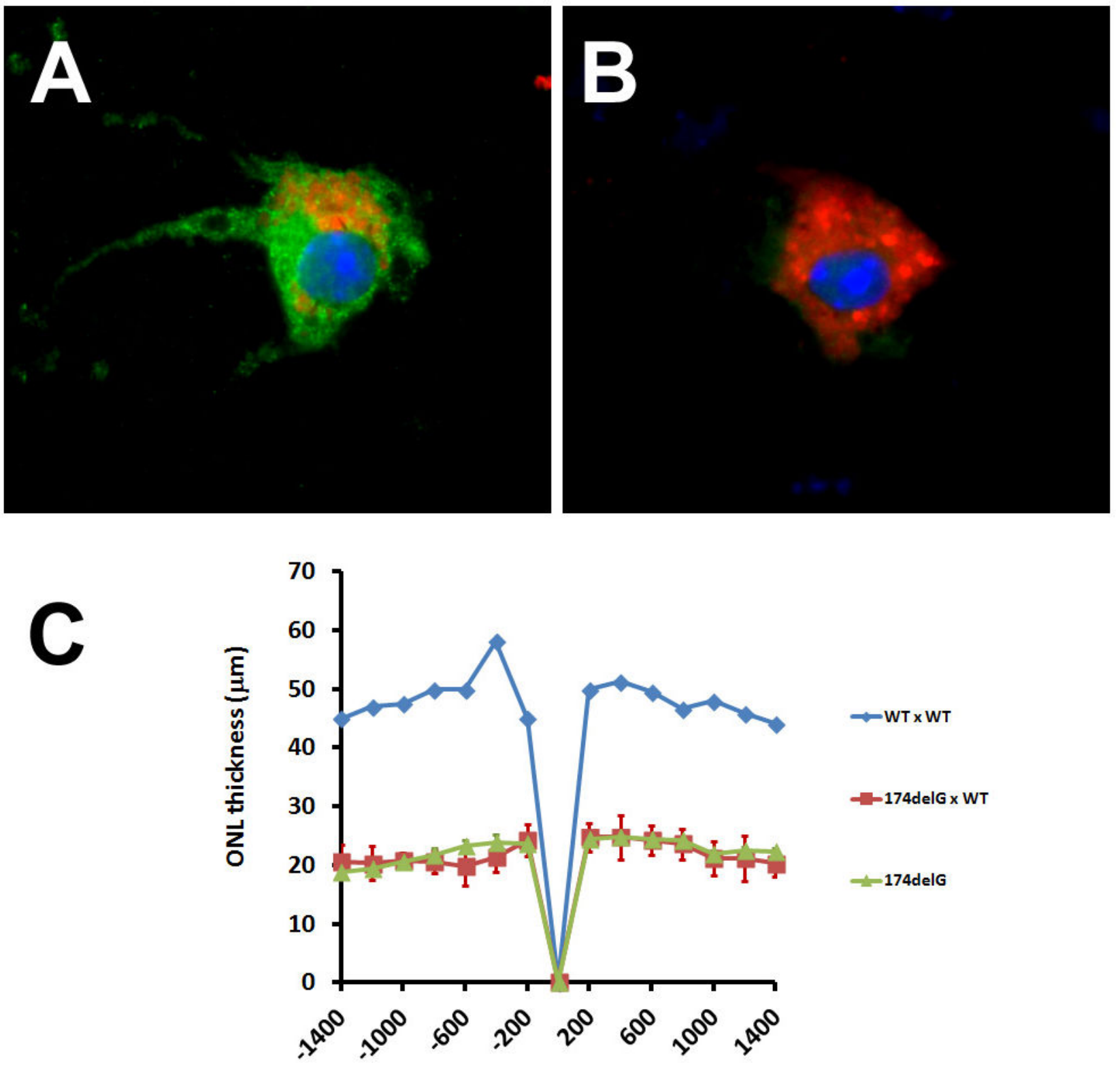

Eccentricity $(\mu \mathrm{m})$

Figure 3. Subretinal cells are derived directly from circulating monocytes and replacing them with wild type cells does not alter disease progression

YFP+ bone marrow was transplanted into irradiated $M$ frp ${ }^{174 d e l G}$ mice at a young age, and then animals were examined several weeks later for evidence of donor cells in the subretinal space. (A) In animals that received a YFP+ transplant, virtually all the subretinal cells contained YFP immunoreactivity. (B) In unaltered Mfrp $^{174 d e l G}$ animals, no immunoreactivity was detected, indicating that the labeling is specific. Red signal is the intrinsic autofluorescence of these cells. They are counterstained with Hoescht to label nuclei (blue). (C) Animals receiving $M f r p^{+/+}$YFP+ bone marrow did not have any significant changes in retinal thickness. 
A

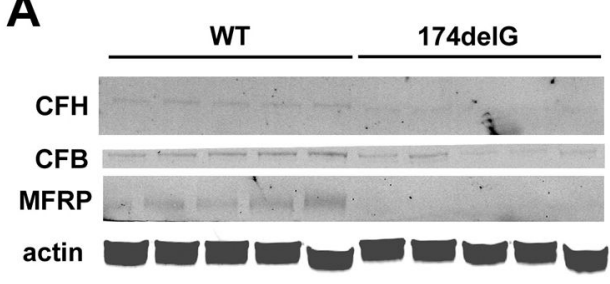

C

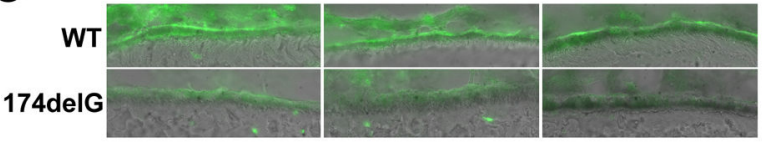

B

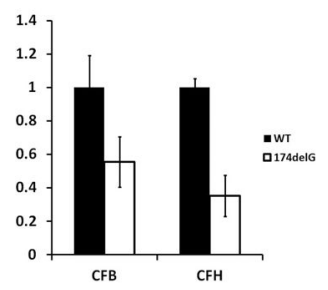

Figure 4. Downregulation of complement factors in $M$ frp ${ }^{174 d e l G}$ RPE+choroid (A) Western analysis of complement factors from RPE+choroid tissue showed a significant decrease in the abundance of CFH and CFB. Western bands are faint because individual $\mathrm{RPE} /$ choroids were used and the amount of material was very limiting, but the bands are accurately quantitated in (B). (C) C3d immunostaining in sections from three different mice of each genotype are shown. The C3d antibody labeled the RPE in both cases, although in wild type animals staining was more intense and concentrated on the basal side of the RPE. 


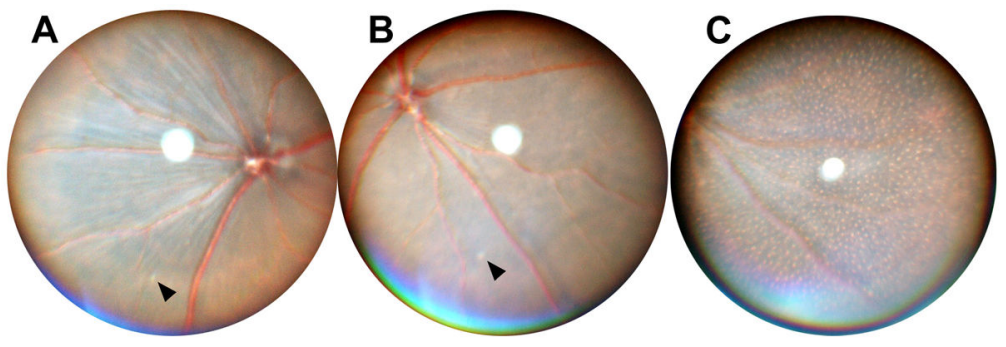

Figure 5. Comparison of $r d 8$ lesions with fundus spots in $M$ frp ${ }^{174 d e l G}$ mice (A-B) Fundus images from 5-month old $r d 8$ mice. Arrows indicate small, discrete spots that are occasionally observed in these animals. (C) Mfrp ${ }^{174 d e l G}$ mice at this age have uniformly small spots with even distribution. 